\title{
Effects of dietary trans-fatty acid isomers on plasma lipoproteins in the Golden Syrian Hamster
}

\author{
Cynthia Tyburczy ${ }^{1}$, Adam Lock ${ }^{2}$, Frederic Destaillats ${ }^{3}$, Dale Bauman ${ }^{1}$ and Andrew Salter ${ }^{4}$ \\ ${ }^{1}$ Cornell University, New York, USA, ${ }^{2}$ University of Vermont, Vermont, USA, ${ }^{3}$ Nestle Research Centre, Lausanne, \\ Switzerland and ${ }^{4}$ University of Nottingham, Nottingham, UK
}

There are two main dietary sources of trans-fatty acids (TFA), those derived from the partial hydrogenation and purification of polyunsaturated oils (PHVO; industrial sources), and those found in the milk and meat of ruminant animals as a result of biohydrogenation of PUFA by rumen bacteria (natural sources). The profile of TFA isomers in foods is markedly different depending on food source, and recent evidence indicates the impact of TFA on the risk of atherosclerosis may also differ according to source ${ }^{(1)}$. Most TFA in the human diet are $\mathrm{C}_{18}$ fatty acids with a single double bond (18:1). Industrial sources of TFA contain a Gaussian distribution of TFA that centres on trans-9 18:1 (elaidic acid; EA) and trans-10 18:1. In milk fat, however, vaccenic acid (trans-11 18:1; VA) is the predominant TFA, typically accounting for $\leq 80 \%$ of the total TFA present. A substantial amount of evidence accumulated over the past two decades points to TFA as being major risk factors for atherosclerosis ${ }^{(2)}$. High intakes have been associated with increased plasma total cholesterol, LDLcholesterol and TAG as well as decreased plasma HDL-cholesterol. However, most of this evidence relates to total TFA intake and only limited data are available concerning the source of TFA or the effects of individual isomers.

In the present experiment male Golden Syrian Hamsters (eight per group) were fed chow-based diets supplemented with $12.5 \%$ fat (formulated to approximate the fatty acid composition of a typical Western' diet) and $0.2 \%$ (w/w) cholesterol for 4 weeks. The diet was further supplemented with $2.5 \%(\mathrm{w} / \mathrm{w})$ PHVO, EA or VA. At the end of the trial plasma lipoproteins were separated by preparative ultracentrifugation and cholesterol content measured enzymically.

Compared with animals consuming PHVO, those fed EA or VA had significantly reduced cholesterol concentrations (mmol/l) in plasma VLDL (2.00 (SD 0.50) v. 1.21 (SD $0.19 ; P=0.006)$ and 1.36 (SD $0.56 ; P=0.037$ ) respectively), IDL (0.39 (SD 0.21$) v .0 .10$ (SD 0.05 ; $P<0.001)$ and 0.16 (SD $0.07 ; P=0.009)$ respectively) and LDL $(1.10(\mathrm{SD} 0.24) v .0 .46$ (SD $0.17 ; P<0.001)$ and 0.76 (SD $0.12 ; P=0.007)$ respectively) fractions, while HDL-cholesterol was not significantly different. As a result, the atherogenic lipoprotein-cholesterol (VLDL + IDL + LDL):anti-atherogenic HDL-cholesterol was reduced from 1.69 (SD 0.35) in the PHVO group to 0.74 (SD 0.11 ) in the EA group $(P<0.001)$ and $1.18(\mathrm{SD} 0.37)$ in the VA group $(P=0.011)$. These data therefore indicate that neither of these two isomers are responsible for the hypercholesterolaemic effects of PHVO.

The effect of PHVO and the two TFA isomers on hepatic lipid-fatty acid composition was also investigated. Consumption of PHVO led to an accumulation of a range of 18:1 trans-isomers with the double bond in positions 6-12, representing a total trans-18:1 content of $1.95 \%$ total fatty acids. Feeding EA and VA led to the accumulation of the two isomers in hepatic lipids $(2.61$ and $2.88 \%$ total fatty acids respectively). VA consumption was also associated with an accumulation of cis-9, trans-11 conjugated linoleic acid (rumenic acid; RA) in liver lipids $(1.08 \%$ total fatty acids compared with 0.28 and $0.18 \%$ total fatty acids $(P<0.001)$ in animals fed PHVO and EA respectively). This finding suggests significant conversion of VA to RA through the action of $\Delta 9$-desaturase in the tissues of VA-fed animals. RA has been associated with a range of potential health benefits, including anti-atherogenic and anti-carcinogenic actions ${ }^{(3)}$.

It is concluded that both EA and VA have similar effects on plasma lipoprotein concentrations and both reduce the concentration of potentially-atherogenic lipoproteins compared with PHVO. This outcome suggests that components of PHVO other than VA and EA must be responsible for its cholesterol-raising properties. This action could relate to other trans-18:1 isomers or one or more of the many other cis- or trans-isomers that are found in small quantities in PHVO. The lack of any cholesterol-raising effects of VA, coupled with its conversion to potentially-beneficial RA within tissues suggests that this TFA, which is the predominant isomer in ruminant meat and milk may actually confer significant health benefits and should be considered separately from other TFA associated with PHVO.

1. Lock AL, Parodi PW \& Bauman DE. (2005) Aust J Dairy Technol 60, 134-142.

2. Stender S \& Dyerberg J (2004) Ann Nutr Metab 48, 61-66.

3. Salter AM, Lock AL, Garnsworthy PC \& Bauman DE (2006) In Recent Advances in Animal Nutrition, pp. 1-18. Nottingham: Nottingham University Press. 